\title{
A remark on nonlinear accessibility conditions and infinite prolongations*
}

\author{
Michel Fliess \\ Laboratoire des Signaux et Systèmes, CNRS-Supélec \\ Plateau de Moulon, 91192 Gif-sur-Yvette, France \\ e-mail: fliess@lss.supelec.fr \\ Jean Lévine, Philippe Martin
}

Centre Automatique et Systèmes, École des Mines de Paris 35 rue Saint-Honoré, 77305 Fontainebleau, France e-mail: $\{$ levine, martin\}@cas .ensmp.fr

François Ollivier GAGE-CNRS, Centre de Mathématiques École polytechnique, 91128 Palaiseau, France e-mail: ollivier@gage.polytechnique.fr

\section{Pierre Rouchon}

Centre Automatique et Systèmes, École des Mines de Paris 60 bld. Saint-Michel, 75272 Paris Cedex 06, France e-mail: rouchon@cas. ensmp.fr

*Work partially supported by the G.D.R. "MEDICIS" of the C.N.R.S. 


\begin{abstract}
The Lie rank condition for strong nonlinear accessibility is interpreted via the differential geometry of jets and prolongations of infinite order. It yields to a new Lie algebraic criterion and to the consideration of first integrals, which apply to nonlinear systems in quite general form; the latter characterization in particular is valid without distinguishing between the system variables and is independent of time-scalings. Weak accessibility is also considered.
\end{abstract}

Keywords: Nonlinear controllability, strong accessibility, weak accessibility, Lie brackets, first integrals, jets, prolongations, diffieties, flatness. 


\section{Introduction}

Modern nonlinear differential geometric control began more than twenty five years ago by trying to extend to nonlinear systems Kalman's controllability (see, e.g., [7, 18, 33, 34, 42, 43, 61, 64]; more details may be found in [39, $47,62])$. We remark here that the strong and weak accessibility Lie rank conditions possess an easy and nice interpretation within the framework of the differential geometry of jets and prolongations of infinite order, which is becoming a mainstay in some parts of mathematics and physics (see, e.g., $[3,4,32,38,41,49,66,67,69]$ and the references therein). Motivated by the study of differential flatness [25], i.e., by dynamic feedback linearizabilty, this infinite-dimensional geometry is now being developed in nonlinear control ( $c f$. $[23,24,27,28,29,48,52])$. Considering those prolongations means taking into account time derivatives of arbitrary order of the system variables. This system theoretic trend, which has also been formalized via differential algebra (cf. $[19,20,22,25]$ ), is apparent, not only in dynamic feedback linearization (see, e.g., [5, 9, 59, 60]), but also with Singh's nonlinear extension [58] of Silverman's structure algorithm; it plays thus a crucial rôle in input-output inversion and in related synthesis problems like dynamic feedback decoupling and perturbation rejection, model matching, etc... (see, e.g., [10, 11, 12, 13, 14, 15, 16, 19, 36, 37, 45, 46, 47, 51, 53, 54, 55, 56]).

Take a dynamics $\dot{x}=F(x, u)$, where $u=\left(u_{1}, \ldots, u_{m}\right)$ and $x=$ $\left(x_{1}, \ldots, x_{n}\right)$ belong to some open subsets of $\mathbb{R}^{m}$ and $\mathbb{R}^{n} ; F=\left(F_{1}, \ldots, F_{n}\right)$ is a smooth $\mathbb{R}^{n}$-valued function of its arguments. The infinite order prolongation is the infinite-dimensional manifold $\mathcal{D}(c f .[32,49])$ described by the coordinates $\left\{t, x_{1}, \ldots, x_{n}, u_{i}^{\left(\nu_{i}\right)} \mid i=1, \ldots, n ; \nu_{i} \geq 0\right\}$, where the total derivative $\frac{d}{d t}$ with respect to the time $t$ is given by the following infinite vector field, called a Cartan field (cf. [41, 69]),

$$
\frac{d}{d t}=\frac{\partial}{\partial t}+\sum_{k=1}^{n} F_{k} \frac{\partial}{\partial x_{k}}+\sum_{i=1}^{m} \sum_{\nu_{i} \geq 0} u_{i}^{\left(\nu_{i}+1\right)} \frac{\partial}{\partial u_{i}^{\left(\nu_{i}\right)}}
$$

The dynamics satisfies locally, i.e., on an open subset, the Lie rank strong accessibility condition due to Sussmann and Jurdjevic [64] if, and only if, one of the two following equivalent conditions is verified:

- The tangent distribution on $\mathcal{D}$ is locally spanned by the vector fields $\left\{\frac{d}{d t}, \frac{\partial}{\partial u_{i}^{\left(\nu_{i}\right)}} \mid i=1, \ldots, m ; \nu_{i} \geq 0\right\}$ and their iterated Lie brackets. 
- The only local first integrals or constants of motion, i.e., local smooth real-valued functions $I$ on $\mathcal{D}$, such that $\frac{d I}{d t}=0$, are trivial, i.e., constant.

The first condition, which is perhaps easier to read than the usual Lie bracket strong accessibility criterion, only necessitates in practice a finite number of finite vector fields, i.e., here $\sum_{k=1}^{n} F_{k} \frac{\partial}{\partial x_{k}}+\sum_{i=1}^{m} \dot{u}_{i} \frac{\partial}{\partial u_{i}}$, and $\frac{\partial}{\partial u_{\iota}}, \frac{\partial}{\partial \dot{u}_{\iota}}, \iota=1, \ldots, m$. It is still valid, without any modification, for timedependent dynamics of the type $\dot{x}=G\left(t, x, u, \dot{u}, \ldots, u^{(\alpha)}\right)$, with derivatives of the control variables, which may appear in practice (see, e.g., [30]). The second characterization, which relates strong accessibility to first integrals and constants of motion, i.e., a classic and important notion in mechanics and physics ( $c f .[2,47,50])$, is independent of any distinction between the system variables. It confirms therefore Willems' standpoint [68], and applies to many realistic case-studies which are written in most general implicit forms, i.e., not necessarily in the classic explicit state-variable representation (see, e.g., $[8,25,30,44]$ ). The second characterization is also independent of time scalings, which is playing a crucial rôle in some feedback stabilization problems (cf. [26]). We thus introduce the notion of diffiety, i.e., of an abstract infinite-dimensional manifold equipped with a Cartan distribution (see $[41,65,66,67,69]$ ), which is justified by an example. We show that an orbitally flat system, which is Lie-Bäcklund isomorphic to a trivial diffiety, does not possess any non-trivial local first integral. The considerations and results above lead us to define local controllability as the absence of any non-trivial local first integral. The proofs are elementary and only require standard facts in differential geometry and control.

The results on weak accessibility bear strong similarities to the previous ones. They are obtained by controlling the clock (cf. [23, 24]) which yields an interesting equivalence between weak and strong accessibilities.

The conclusion lists a further extension and some open questions in this infinite-dimensional setting. 


\section{$1 \quad$ Strong accessibility}

1.1. Consider the control-affine dynamics

$$
\dot{x}=f_{0}(x)+\sum_{i=1}^{m} u_{i} f_{i}(x)
$$

where the state $x=\left(x_{1}, \ldots, x_{n}\right)$ and the control $u=\left(u_{1}, \ldots, u_{m}\right)$ belong to open subsets of $\mathbb{R}^{n}$ and $\mathbb{R}^{m} ; f_{j}=\left(f_{j, 1}, \ldots, f_{j, n}\right), j=0,1, \ldots, m$, is a $\mathbb{R}^{n}$ valued $C^{\infty}$-function of $x$, which we consider as a vector field (cf. [39, 47]), i.e., $f_{j}=f_{j, 1} \frac{\partial}{\partial x_{1}}+\ldots+f_{j, n} \frac{\partial}{\partial x_{n}}$. Let $L$ be the weak accessibility distribution, i.e., the distribution spanned by the Lie algebra $\mathcal{L}$ generated by $f_{0}, f_{1}, \ldots, f_{m} ; L_{0}$ is the strong accessibility distribution, i.e., the distribution spanned by the Lie ideal $\mathcal{L}_{0}$ in $\mathcal{L}$ generated by $f_{1}, \ldots, f_{m}$. Dynamics $(1)$ is said to satisfy locally ${ }^{1}$ the strong accessibility Lie rank condition [64] (see, also, [35, 39, 57]), if, and only if, $L_{0}$ is locally of dimension $n$.

Take now the nonlinear dynamics

$$
\dot{x}=F(x, u)
$$

where the state $x=\left(x_{1}, \ldots, x_{n}\right)$ and the control $u=\left(u_{1}, \ldots, u_{m}\right)$ belong to open subsets of $\mathbb{R}^{n}$ and $\mathbb{R}^{m} ; F=\left(F_{1}, \ldots, F_{n}\right)$ is a $m$-tuple of $C^{\infty}$-functions of their arguments. It is easy to verify (cf. [57]) that (2) satisfies locally the strong accessibility Lie rank condition if, and only if, the following extended control-affine dynamics shares the same property:

$$
\begin{gathered}
\dot{x}=F(x, y) \\
\dot{y}_{i}=v_{i} \\
i=(1, \ldots, m)
\end{gathered}
$$

where $y=\left(y_{1}, \ldots, y_{m}\right)$; the state $\left(x_{1}, \ldots, x_{n}, y_{1}, \ldots, y_{m}\right)$ and the control $\left(v_{1}, \ldots, v_{m}\right)$ of $(3)$ belong to open subsets of $\mathbb{R}^{n+m}$ and $\mathbb{R}^{m}$.

1.2. Associate to $(2)$ the infinite-dimensional manifold $\mathcal{D}$ given by the local coordinates $\left\{t, x_{1}, \ldots, x_{n}, u_{i}^{\left(\nu_{i}\right)} \mid i=1, \ldots, n ; \nu_{i} \geq 0\right\}^{2}$. The natural topology

\footnotetext{
${ }^{1} \mathrm{~A}$ property is said to hold locally if it holds on an open subset. A local function is a function which is defined locally, i.e., on an open subset.

${ }^{2}$ The necessity of including the time $t$ for the time-invariant system (2) will become clear in the next section when considering first integrals.
} 
on $\mathcal{D}$ is Fréchet (cf. [32]): open subsets of $\mathcal{D}$ are defined by the fact that a finite number of the variables among $\left\{t, x_{1}, \ldots, x_{n}, u_{i}^{\left(\nu_{i}\right)}\right\}$ belong to open subsets of $\mathbb{R}$, the others being arbitrary. A local real-valued $C^{\infty}$-function on $\mathcal{D}$ is a local real-valued $C^{\infty}$-function which depends only on a finite number of variables among $\left\{t, x_{1}, \ldots, x_{n}, u_{i}^{\left(\nu_{i}\right)}\right\}$. Define on $\mathcal{D}$ the infinite vector field

$$
\frac{d}{d t}=\frac{\partial}{\partial t}+\sum_{k=1}^{n} F_{k} \frac{\partial}{\partial x_{k}}+\sum_{i=1}^{m} \sum_{\nu_{i} \geq 0} u_{i}^{\left(\nu_{i}+1\right)} \frac{\partial}{\partial u_{i}^{\left(\nu_{i}\right)}}
$$

It is denoted $\frac{d}{d t}$ since when applied to a local real-valued $C^{\infty}$-function $h\left(t, x, u, \dot{u}, \ldots, u^{(\nu)}\right)$ on $\mathcal{D}$ it yields its total derivative with respect to $t: \frac{d}{d t}$ is called a Cartan field (cf. [41, 69]). The tangent distribution of $\mathcal{D}$ is spanned by $\frac{\partial}{\partial t}, \frac{\partial}{\partial x_{1}}, \ldots, \frac{\partial}{\partial x_{n}}, \frac{\partial}{\partial u_{i}^{\left(\nu_{i}\right)}}$.

1.3. A local first integral, or constant of motion, $I$ on $\mathcal{D}$ is a local real-valued $C^{\infty}$-function $I$ on $\mathcal{D}$ such that $\frac{d I}{d t}=0$ (compare with $[2,47,50]$ ). It is called trivial if the function $I$ is a constant.

1.4. Theorem. For dynamics (2) the three following properties are equivalent:

(i) (2) satisfies locally the strong accessibility Lie rank condition.

(ii) The tangent distribution of $\mathcal{D}$ is locally spanned by the Lie algebra generated by $\left\{\frac{d}{d t}, \frac{\partial}{\partial u_{i}^{\left(\nu_{i}\right)}} \mid i=1, \ldots, m ; \nu_{i} \geq 0\right\}$.

(iii) The vector fields $\left\{\frac{\partial}{\partial x_{i}} \mid i=1, \ldots n\right\}$ belong locally to the Lie algebra generated by $\left\{\frac{d}{d t}, \frac{\partial}{\partial u_{i}^{\left(\nu_{i}\right)}} \mid i=1, \ldots, m ; \nu_{i} \geq 0\right\}$.

(iv) The only local first integrals on $\mathcal{D}$ are the trivial ones.

1.5. Proof. It follows from section 1.1 that we may restrict ourselves to (1), i.e., to the control-affine case $F(x, u)=f_{0}(x)+\sum_{i=1}^{m} u_{i} f_{i}(x)$. Straightforward Lie bracket calculations yield

$$
\left[\frac{\partial}{\partial u_{i}^{\left(\nu_{i}\right)}}, \frac{d}{d t}\right]=\left\{\begin{array}{l}
f_{i} \quad \text { if } \nu_{i}=0 \\
\frac{\partial}{\partial u_{i}^{\left(\nu_{i}-1\right)}} \quad \text { if } \nu_{i} \geq 1
\end{array}\right.
$$

and

$$
\left[\frac{d}{d t}, f_{i}\right]=\left[f_{0}, f_{i}\right]+\sum_{j=1}^{m} u_{j}\left[f_{j}, f_{i}\right]
$$


It follows at once that $(i i)$ and (iii) are satisfied if, and only if, $L_{0}$ is locally of dimension $n$. Thus, $(i) \Longleftrightarrow($ ii $) \Longleftrightarrow($ iii $)$.

Assume that (1) does not locally satisfy the strong accessiblity Lie rank condition. Then (1) may be locally decomposed into (see, e.g., [39, 47]):

$$
\begin{gathered}
\dot{\bar{x}}=\bar{f}_{0}(\bar{x}) \\
\dot{\tilde{x}}=\tilde{f}_{0}(\bar{x}, \tilde{x})+\sum_{i=1}^{m} u_{i} \tilde{f}_{i}(\bar{x}, \tilde{x})
\end{gathered}
$$

where $\bar{x}=\left(\bar{x}_{1}, \ldots, \bar{x}_{k}\right)$ and $\tilde{x}=\left(\tilde{x}_{k+1}, \ldots, \tilde{x}_{n}\right)$. Applying the classic straightening-out theorem (see, e.g., [2]) to $\dot{\bar{x}}=\bar{f}_{0}(\bar{x})$ yields locally $\dot{z}_{1}=$ $1, \dot{z}_{2}=0, \ldots, \dot{z}_{k}=0$. We get $k$ non-trivial first integrals $z_{1}-t, z_{2}, \ldots, z_{k}$. Let us now assume the existence of a non-trivial first integral $I\left(t, x, u, \dot{u}, \ldots, u^{(\alpha)}\right)$ such that, for some integer $i \in\{1, \ldots, m\}, \frac{\partial I}{\partial u_{i}^{(\alpha)}} \neq 0$. Then $\frac{d I}{d t}$ contains the term $u_{i}^{(\alpha+1)} \frac{\partial I}{\partial u_{i}^{(\alpha)}}$; such an affine function of $u_{i}^{(\alpha+1)}$ may be identically zero if, and only if, $\frac{\partial I}{\partial u_{i}^{(\alpha)}}$ is so. An immediate induction shows that any first integral depends only on $t, x_{1}, \ldots, x_{n}$. The existence of such a local non-trivial first integral clearly contradicts (iii). Thus, $(i i i) \Longleftrightarrow(i v)$. (Q.E.D.)

1.6. Take a time-dependent dynamics

$$
\dot{x}=G\left(t, x, u, \dot{u}, \ldots, u^{(\alpha)}\right)
$$

which may contain moreover derivatives of the control variables (cf. [30]) and an analogous infinite-dimensional manifold $\mathcal{E}$ given by the local coordinates $\left\{t, x_{1}, \ldots, x_{n}, u_{i}^{\left(\nu_{i}\right)}\right\}$ and

$$
\frac{d}{d t}=\frac{\partial}{\partial t}+\sum_{k=1}^{n} G_{k} \frac{\partial}{\partial x_{k}}+\sum_{i=1}^{m} \sum_{\nu_{i} \geq 0} u_{i}^{\left(\nu_{i}+1\right)} \frac{\partial}{\partial u_{i}^{\left(\nu_{i}\right)}}
$$

where $G=\left(G_{1}, \ldots, G_{n}\right)$. Associate to $(5)$ the control-affine system

$$
\begin{gathered}
\dot{x}_{0}=1 \\
\dot{x}=G\left(x_{0}, x, y^{0}, y^{1}, \ldots, y^{\alpha}\right) \\
\dot{y}_{i}^{0}=y_{i}^{1} \\
\ldots \\
\dot{y}_{i}^{\alpha-1}=y_{i}^{\alpha} \\
\dot{y}_{i}^{\alpha}=v_{i} \\
(i=1, \ldots, m)
\end{gathered}
$$


where $y^{\mu}=\left(y_{1}^{\mu}, \ldots, y_{m}^{\mu}\right), \mu=1, \ldots, \alpha$. We obtain at once a similar result to the theorem in section 1.5.

Proposition. For dynamics (5) the two following properties are equivalent: (i) The tangent distribution of $\mathcal{E}$ is locally spanned by the Lie algebra generated by $\left\{\frac{d}{d t}, \frac{\partial}{\partial u_{i}^{\left(\nu_{i}\right)}} \mid i=1, \ldots, m ; \nu_{i} \geq 0\right\}$.

(ii) The only local first integrals on $\mathcal{E}$ are the trivial ones.

A dynamics (5) which (locally) satisfies one of the two preceeding equivalent conditions will be called (locally) controllable, or strongly accessible.

1.7. REmark. By taking advantage of (3), property $(i i)$ in the above theorem may be checked by looking at the Lie algebra generated by $\sum_{k=1}^{n} F_{k} \frac{\partial}{\partial x_{k}}+$ $\sum_{i=1}^{m} \dot{u}_{i} \frac{\partial}{\partial u_{i}}, \frac{\partial}{\partial u_{\iota}}$ and $\frac{\partial}{\partial u_{\iota}}, \iota=1, \ldots, m$. We thus only need usual vector fields, i.e., vector fields which are given by finite expressions and not infinite ones. From (6) the same conclusion holds for property $(i)$ in the above proposition. 1.8. REMARK. Controllability is usually related to some reachability properties in the state-space (see, e.g., [39, 47, 62] and the references therein) which might be misleading in the presence of control derivatives. Take, for instance, the one-dimensional single-input linear dynamics $\dot{x}=\dot{u}$. It is uncontrollable since $x-u$ is a non-trivial first integral ${ }^{3}$. The equality $x(t)=u(t)+$ const. demonstrates however that from any point in the state-space $\mathbb{R}$ any other one may be reached in an arbitrary small time.

1.9. In many practical situations systems are not given a priori as a dynamics (2) even in the generalized form (5) (see, e.g., [8, 30, 44]). It might even be unwise to try to write them so as shown by the example in section 1.11 below ${ }^{4}$. We introduce therefore a $C^{\infty}$-manifold $\mathcal{M}$ which is modelled not necessarily on $\mathbb{R}^{n}$, where $n$ is finite, but also on $\mathbb{R}^{\infty}$, i.e., on an infinite, but denumerable, Cartesian product $\mathbb{R} \times \mathbb{R} \times \ldots \times \mathbb{R} \times \ldots$ of copies of $\mathbb{R}$. As already said in section 1.2, the natural topology of $\mathcal{M}$ is Fréchet. A local realvalued $C^{\infty}$-function on $\mathcal{M}$ is defined in a local chart as an usual $C^{\infty}$-function of a finite number of coordinates. A diffiety ${ }^{5}$ is such a manifold $\mathcal{M}$, which is moreover equipped with an involutive distribution, the Cartan distribution, of finite dimension, called its Cartan dimension (see [41, 65, 66, 67, 69] for

\footnotetext{
${ }^{3}$ Notice that Willems' trajectory characterization [68] leads to the same conclusion, as well as its module-theoretic counterpart in [21].

${ }^{4}$ Another more realistic case-study is the crane considered in [30] and [25].

${ }^{5}$ This terminology is due to Vinogradov [66], who certainly is the main contributor to this new and fascinating area.
} 
more details). A local vector field, which is a local section of the Cartan distribution, is called a Cartan field. All our diffieties will be ordinary, i.e., with Cartan dimension one. Examples of ordinary diffieties are $\mathcal{D}$ in section 1.2 and $\mathcal{E}$ in section 1.6; in both cases the Cartan distributions are spanned by $\frac{d}{d t}$. In this setting a system $S$ is nothing else than an ordinary diffiety ${ }^{6}$. A (local) trajectory of the system is in this setting a (local) integral curve on the diffiety of a Cartan field (compare with Willems' behavioral approach [68]).

A local first integral, or a constant of motion, on $S$ is a local real-valued $C^{\infty}$-function $I$ on $S$ such that its Lie derivative with respect to a, and therefore any, Cartan field is identically zero. It is said to be trivial if it is a constant.

Definition. The system $S$ is said to be locally controllable, or strongly accessible, if, and only if, any local first integral is trivial.

1.10. Remark. This latter definition, which is coordinate-free, is clearly independent of any distinction between system variables and therefore agrees with Willems' viewpoint [68]. It is also independent of time-scalings, which may be understood in this setting as choosing two different Cartan fields, like in $\mathcal{D}$ or $\mathcal{E}, \frac{d}{d t}$ and $\gamma \frac{d}{d t}$, where $\gamma \neq 0$ is a local real-valued $C^{\infty}$-function.

1.11. EXAMPLE. Consider the implicit system

$$
\begin{aligned}
\dot{x}+\dot{y} & =u \\
x+y^{2} & =u
\end{aligned}
$$

where $x, y, u \in \mathbb{R}$. Obvious calculations yield the explicit generalized dynamics

$$
\dot{y}=\frac{u-\dot{u}}{1-2 y}
$$

which is not defined for $y=\frac{1}{2}$. On the other hand, the diffiety associated to (7) ${ }^{7}$ is given by the global coordinates $\left\{t, x, y^{(\nu)} \mid \nu \geq 0\right\}$. Its Cartan distribution is spanned by the Cartan field

$$
\frac{d}{d t}=\frac{\partial}{\partial t}+\left(x+y^{2}-\dot{y}\right) \frac{\partial}{\partial x}+\sum_{\nu \geq 0} y^{(\nu+1)} \frac{\partial}{\partial y^{(\nu)}}
$$

\footnotetext{
${ }^{6}$ Compare with [28] where the notion of diffiety of finite type is utilized.

${ }^{7}$ Determining the diffiety from the given equations may not be always as straightforward as here. See [49] and [69] for details.
} 
The reader will easily check that (7) is locally controllable.

1.12. ExAmPle. The trivial diffiety (cf. $[65,69]$ ), or trivial system, $\mathbb{R} \times \mathbb{R}_{m}^{\infty}$ is given by the global coordinates $\left\{t, y_{i}^{\left(\nu_{i}\right)} \mid i=1, \ldots, m ; \nu_{i} \geq 0\right\}$; its Cartan distribution is spanned by

$$
\frac{d}{d t}=\frac{\partial}{\partial t}+\sum_{i=1}^{m} \sum_{\nu_{i} \geq 0} y_{i}^{\left(\nu_{i}+1\right)} \frac{\partial}{\partial y_{i}^{\left(\nu_{i}\right)}}
$$

It is called trivial since it corresponds to the trivial equation $0=0$.

1.13. The following result is already known in the framework of homological algebra, i.e., via Vinogradov's variational bicomplex (see, e.g., [49, 65, 69] and the references therein $)^{8}$. Our proof below is completely elementary ${ }^{9}$. Proposition. $\mathbb{R} \times \mathbb{R}_{m}^{\infty}$ is locally controllable.

Proof. Take a local first integral $I\left(t, y, \ldots, y^{(\alpha)}\right)$ where, like in section 1.5, for some integer $i \in\{1, \ldots, m\}, \frac{\partial I}{\partial y_{i}^{(\alpha)}} \neq 0$. Then $\frac{d I}{d t}$, which contains the term $y_{i}^{(\alpha+1)} \frac{\partial I}{\partial y_{i}^{(\alpha)}}$, can be identically zero if, and only if, $\frac{\partial I}{\partial y_{i}^{(\alpha)}}$ is so. By induction, $I$ depends only on $t$. The equality $\frac{\partial I}{\partial t}=0$ proves that $I$ is trivial. (Q.E.D.)

1.14. A local Lie-Bäcklund mapping ${ }^{10}$ between two diffieties is a local $C^{\infty}$ mapping which is compatible with the Cartan distributions (cf. [69]). The notion of local Lie-Bäcklund isomorphism is clear and leads to a most interesting notion of equivalence between systems (see [27, 28, 29]). A system is said to be orbitally flat $[27,28,29]$ if, and only if, it is locally Lie-Bäcklund isomorphic to a trivial system $\mathbb{R} \times \mathbb{R}_{m}^{\infty}{ }^{11}$. The next result follows at once from section 1.13 .

COROLlary. An orbitally flat system is locally controllable ${ }^{12}$.

\footnotetext{
${ }^{8}$ Notice that the existence of first integrals on general diffieties may be interpreted in this homological framework.

${ }^{9}$ Its generalization to the partial case, i.e., to the case where the Cartan dimension is strictly greater than 1 , is immediate.

${ }^{10}$ This terminology, which is borrowed from Ibragimov [4, 38] (see, also, [69]), is quite popular in mathematical physics, although it is heavily criticized by some authors, like Olver [49]. Vinogradov and his school (see [41, 66, 67]), are writing $C$-mappings, where $C$ stands for contact.

${ }^{11}$ This notion is slightly more general than differential flatness [25, 27, 28, 29, 48, 52], where time is preserved. See [25, 26, 27, 28, 29] for some applications of flatness and, also, [1].

${ }^{12}$ See $[27,29]$ for another approach to the reachability property of differentially flat systems.
} 


\section{Weak accessibility}

2.1. Recall (cf. [39, 47]) that (1) is said to satisfy locally the weak accessibility Lie rank condition if, and only if, the weak accessibility distribution $L$, defined in section 1.1, is locally of dimension $n$. Standard Lie bracket calculations demonstrate again that (2) satisfies locally the weak accessibility condition if, and only if, (3) does so.

2.2. Associate to (2) the dynamics

$$
\dot{x}=v F(x, u)
$$

where $v \in \mathbb{R}$ is a new one-dimensional control variable ${ }^{13}$. Since (8) is driftless, standard Lie bracket calculation lead to the

LEMmA. (2) satisfies locally the weak accessibility Lie rank condition if, and only if, (8) satisfies locally the strong accessibility Lie rank condition.

2.3. THEOREM. For dynamics (2), the four following propositions are equivalent:

(i) (2) is locally weakly accessible.

(ii) The tangent distribution of $\mathcal{D}$ is locally spanned by the Lie algebra generated by $\left\{\frac{d}{d t}, \frac{\partial}{\partial t}, \frac{\partial}{\partial u_{i}^{\left(\nu_{i}\right)}} \mid i=1, \ldots, m ; \nu_{i} \geq 0\right\}$.

(iii) The vector fields $\left\{\frac{\partial}{\partial x_{i}} \mid i=1, \ldots n\right\}$ belong locally to the Lie algebra generated by $\left\{\frac{d}{d t}, \frac{\partial}{\partial t}, \frac{\partial}{\partial u_{i}^{\left(\nu_{i}\right)}}\right\}$.

(iv) There exists, up to functional independence ${ }^{14}$, at most one non-trivial local first integral, which is then necessarily time-dependent.

ProOF. Like in section 1.5, we may restrict ourselves to (1). Standard Lie bracket calculations yield, like in section 1.5 , the equivalence $(i) \Longleftrightarrow$ $($ ii $) \Longleftrightarrow($ iii $)$.

Assume that (1) satisfies locally the weak accessibility Lie rank condition but not the strong one. Combining in the decomposition (4) the straightening-out theorem and the lemma of section 2.2 shows that $k=1$ and $z_{1}=1$. It yields the time-dependent first integral $z_{1}-t$. Thus, $(i) \Longleftrightarrow(i v)$. (Q.E.D.)

\footnotetext{
${ }^{13}$ Set $d t=v d \tau$. This control of the clock [23, 24] yields indeed $d x / d \tau=v F(x, u)$.

${ }^{14}$ See, e.g., [49] for this classic notion.
} 


\section{Conclusion}

A future publication will relate the strong accessibility condition to the Lie algebra of generalized symmetries, which is known to play a prominent rôle in several questions of mathematical physics (see, e.g., [41, 49, 65, 67, 69] and the references therein). We also suggest that the language and the techniques from jets and prolongations of infinite order may shed some light on one of the most interesting research area in nonlinear control, namely local controllability (see, e.g., [6, 40, 63]). As a matter of fact, local controllability and optimal control are on one hand intimately related; variational calculus on the other hand is known to interact strongly with infinite prolongations (see, e.g., [49, 69] and the references therein).

Observability will be studied elsewhere (see [17, 31] for first hints).

\section{References}

[1] E.A. Abbott, Flatland (Anatolia, Paris, 1996).

[2] R. Abraham, J.E. Marsden and T. Ratiu, Manifolds, Tensor Analysis, and Applications, $2^{\text {nd }}$ ed. (Springer, New York, 1988).

[3] D.V. Alekseevskij, A.M. Vinogradov and V.V. Lychagin, Geometry I, Basic Ideas and Concepts from Differential Geometry (translated from the Russian), Encycl. Math. Sci. 28 (Springer, Berlin, 1991).

[4] R.L. Anderson and N.H. Ibragimov, Lie-Bäcklund Transformations in Applications (SIAM, Philadelphia, 1979).

[5] E. Aranda-Bricaire, C.H. Moog, and J.-B. Pomet, A linear algebraic framework for dynamic feedback linearization, IEEE Trans. Automat. Control, 40 (1995) 127-132.

[6] R.M. Bianchini and G. Stefani, Controllability along a trajectory: a variational approach, SIAM J. Control Optimiz. 31 (1993) 900-927.

[7] R.W. Brockett, System theory on group manifolds and coset spaces, SIAM J. Control, 10 (1972) 265-284.

[8] S.L. Campbell, High index differential algebraic equations, J. Mechanics Structures Machines, 23 (1995) 199-222. 
[9] B. Charlet, J. Lévine, and R. Marino, Sufficient conditions for dynamic state feedback linearization, SIAM J. Control Optimiz., 29 (1991) 38-57.

[10] E. Delaleau, Sur la dérivée de l'entrée en représentation et commande des systèmes non linéaires, Thèse (Université Paris-Sud, Orsay, 1993).

[11] E. Delaleau and M. Fliess, Algorithme de structure, filtrations et découplage, C.R. Acad. Sci. Paris, I-315, 1992, pp. 101-106.

[12] E. Delaleau and P.S. Pereira da Silva, Conditions de rang pour le rejet dynamique de perturbations, C.R. Acad. Sci. Paris, I-319, 1994, pp. 1121-1126.

[13] E. Delaleau and P.S. Pereira da Silva, =46iltrations in feedback synthesis, Forum Math. (to appear).

[14] J. Descusse and C.H. Moog, Decoupling with dynamic compensation for strong invertible affine non-linear control systems, Internat. J. Control, 42 (1985) 1387-1398.

[15] J. Descusse and C.H. Moog, Dynamic decoupling for right invertible nonlinear systems, Systems Control Lett., 8 (1987) 345-349.

[16] M.D. DiBenedetto, J.W. Grizzle and C.H. Moog, Rank invariants of nonlinear systems, SIAM J. Control Optimiz., 27 (1989) 658-672.

[17] S. Diop and M. Fliess, On nonlinear observability, Proc. $1^{\text {st }}$ Europ. Control Conf., Grenoble, 1991, pp. 152-157.

[18] D.J. Elliott, A consequence of controllability, J. Diff. Equations, 10 (1970) 364-370.

[19] M. Fliess, Automatique et corps différentiels, =46orum Math., 1 (1989) 227238.

[20] M. Fliess, Generalized controller canonical forms for linear and nonlinear dynamics, IEEE Trans. Automat. Control, 35 (1990) 994-1001.

[21] M. Fliess, A remark on Willems' trajectory characterization of linear controllability, Systems Control Lett., 19 (1992) 43-45.

[22] M. Fliess and S.T. Glad, An algebraic approach to linear and nonlinear control, in Essays on Control: Perspectives in the Theory and its Applications, H. L. Trentelman and J.C. Willems Eds, pp. 223-267 (Birkhäuser, Boston, 1993). 
[23] M. Fliess, J. Lévine, P. Martin and P. Rouchon, Linéarisation par bouclage dynamique et transformations de Lie-Bäcklund, C.R. Acad. Sci. Paris, I-317 (1993) 981-986.

[24] M. Fliess, J. Lévine, P. Martin and P. Rouchon, Nonlinear control and LieBäcklund transformations: towards a new differential geometric standpoint, Proc. $33^{\text {rd }}$ IEEE Decision Control Conf., Lake Buena Vista, FL, 1994, pp. 981-986.

[25] M. Fliess, J. Lévine, P. Martin and P. Rouchon, Flatness and defect of nonlinear systems: introductory theory and applications, Internat. J. Control, 61 (1995) 1327-1361.

[26] M. Fliess, J. Lévine, P. Martin and P. Rouchon, Design of trajectory stabilizing feedback for driftless flat systems, Proc. $3^{\text {rd }}$ European Control Conf., Rome, 1995, pp. 1882-1887.

[27] M. Fliess, J. Lévine, P. Martin and P. Rouchon, A Lie-Bäcklund approach to dynamic feedback equivalence and flatness, in Robust Control via Variable Structure and Lyapunov Techniques, F. Garofalo and L. Glielmo Eds, Lect. Notes Control Inform. Sci. 217, pp. 245-268 (Springer, London, 1996).

[28] M. Fliess, J. Lévine, P. Martin and P. Rouchon, Deux applications de la géométrie locale des diffiétés, Ann. Inst. H. Poincaré Phys. Théor. (1996).

[29] M. Fliess, J. Lévine, P. Martin and P. Rouchon, A Lie-Bäcklund approach to equivalence and flatness of nonlinear systems, (to appear).

[30] M. Fliess, J. Lévine and P. Rouchon, A generalised state variable representation for a simplified crane description, Internat. J. Control, 58 (1993) 277-283.

[31] M. Fliess and J. Rudolph, Corps de Hardy et observateurs asymptotiques locaux pour systèmes différentiellement plats, C.R. Acad. Sci. Paris, (to appear).

[32] M. Golubitsky and V. Guillemin, Stable Mappings and their Singularities (Springer, New York, 1973).

[33] G.W. Haynes and H. Hermes, Nonlinear controllability via Lie theory, SIAM J. Control, 8 (1970) 450-460. 
[34] R. Hermann, On the accessibility problem in control theory, in Inter. Symp. Nonlinear Differential Equations Nonlinear Mechanics, pp. 325-332 (Academic Press, New York, 1963).

[35] R. Hermann and A.J. Krener, Nonlinear controllability and observability, IEEE Trans. Automat. Control, 22 (1977) 728-740.

[36] H.J.C. Huijberts, A nonregular solution of the nonlinear dynamic disturbance decoupling problem with an application to a complete solution of the nonlinear model matching problem, SIAM J. Control Optimiz., 30 (1992) 350-366.

[37] H.J.C. Huijberts, H. Nijmeijer and L.L.M. van der Wegen, Dynamic disturbance decoupling for nonlinear systems, SIAM J. Control Optimiz., 30 (1992) 336-349.

[38] N.H. Ibragimov, Transformation Groups Applied to Mathematical Physics (Reidel, Dordrecht, 1985).

[39] A. Isidori, Nonlinear Control Systems, $3^{\text {nd }}$ ed. (Springer, New York, 1995).

[40] M. Kawski, High order small time local controllability, in Nonlinear Controllability and Optimal Control, H.J. Sussmann Ed., pp. 431-467 (Marcel Dekker, New York, 1990).

[41] I.S. Krasil'shchik, V.V. Lychagin and A.M. Vinogradov, Geometry of Jet Spaces and Nonlinear Partial Differential Equations (Gordon and Breach, New York, 1986).

[42] A.J. Krener, A generalization of Chow's theorem and the bang-bang theorem to nonlinear control systems, SIAM J. Control, 12 (1974) 43-52.

[43] C. Lobry, Contrôlabilité des systèmes non linéaires, SIAM J. Control, 8 (1970) 573-605.

[44] P.J. McLellan, A differential-algebraic perspective on nonlinear controller design methodologies, Chemical Engin. Sci., 49 (1994) 1663-1679.

[45] C.H. Moog, Nonlinear decoupling and structure at infinity, Math. Control Signal Systems, 1 (1988) 257-266.

[46] H. Nijmeijer and W. Respondek, Dynamic input-output decoupling of nonlinear control systems, IEEE Trans. Auyomat. Control, 33 (1988) 1065-1070. 
[47] H. Nijmeijer and A.J. van der Schaft, Nonlinear Dynamical Control Systems (Springer, New York, 1990).

[48] M. van Nieuwstadt, M. Ratinam and R.M. Murray, Differential flatness and absolute equivalence, Proc. $33^{\text {rd }}$ IEEE Conf. Decision Control, Lake Buena Vista, FL, 1994, pp. 326-332.

[49] P.J. Olver, Applications of Lie Groups to Differential Equations, $2^{\text {nd }}$ ed. (Springer, New York, 1993).

[50] P.J. Olver, Equivalence, Invariants, and Symmetry (Cambridge University Press, Cambridge, 1995).

[51] P.S. Pereira da Silva, On the nonlinear dynamic disturbance decoupling problem, J. Math. Systems Estim. Control, 6 (1996) 1-26.

[52] J.-B. Pomet, A differential geometric setting for dynamic equivalence and dynamic linearization, in Geometry in Nonlinear Control and Differential Inclusions, B. Jakubczyk, W. Respondek and T. Rzeżuchowski Eds, pp. 319339 (Banach Center Publications, Warsaw, 1995).

[53] W. Respondek, Right and left invertibility of nonlinear control systems, in Nonlinear Control Theory, H.J. Sussmann Ed., pp. 133-176 (Marcel Dekker, New York, 1990).

[54] J. Rudolph, Viewing input-output system equivalence from differential algebra, J. Math. Systems Estim. Control, 4 (1994) 353-383.

[55] J. Rudolph, Well-formed dynamics under quasi-static state feedback, in Geometry in Nonlinear Control and Differential Inclusions, B. Jakubczyk, W. Respondek and T. Rzeżuchowski Eds, pp. 349-360 (Banach Center Publications, Warsaw, 1995).

[56] J. Rudolph and E. Delaleau, Some examples and remarks on quasi-static feedback of generalized states, (to appear).

[57] A.J. van der Schaft, Observability and controllability for smooth nonlinear systems, SIAM J. Control Optimiz., 20 (1982) 338-354.

[58] S.N. Singh, A modified for invertibility in nonlinear algorithm, IEEE Trans Automat. Control, 26 (1981) 595-598. 
[59] W.M. Sluis, A necessary condition for dynamic feedback linearization, Systems Control Lett., 21 (1993) 277-283.

[60] W.M. Sluis and D.M. Tilbury, A bound on the number of integrators needed to linearize a control systems, Systems Control Lett., (to appear).

[61] H.J. Sussmann, Orbits of families of vector fields and integrability of distributions, Trans. Amer. Math. Soc., 180 (1973) 171-188.

[62] H.J. Sussmann, Lie brackets, real analyticity and geometric control, in Differential Geometric Control Theory, R.W. Brockett, R.S. Millman and H.J. Sussmann Eds, pp. 1-116 (Birkhäuser, Boston, 1983).

[63] H.J. Sussmann, A general theorem on local controllability, SIAM J. Control Optimiz., 25 (1987) 158-194.

[64] H.J. Sussmann and V. Jurdjevic, Controllability of nonlinear systems, J. Differential Equations, 12 (1972) 95-116.

[65] T. Tsujishita, Formal geometry of systems of differential equations, Sugaku Expos., 3 (1990) 25-73.

[66] A.M. Vinogradov, Local symmetries and conservation laws, Acta Appl. Math., 2, 1984, pp. 21-78.

[67] A.M. Vinogradov (Ed.), Symmetries of Partial Differential Equations, Kluwer, Dordrecht, 1989 (reprinted from Acta Appl. Math., 15 (1989) $n^{\circ} 1-2$ \& $\left.16(1989) n^{\circ} 1-2\right)$

[68] J.C. Willems, Paradigms and puzzles in the theory of dynamical systems, IEEE Trans. Automat. Control, 36 (1992) 259-294.

[69] V.V. Zharinov, Geometrical Aspects of Partial Differential Equations (World Scientific, Singapore, 1992). 